\title{
Multi-vector energy optimization tools for energy islands
}

\author{
Sanket Puranik, Heidi Tuiskula, Iliana Ilieva \\ Research and Innovation, \\ Smart Innovation Norway \\ Halden, Norway \\ \{ sanket.puranik, heidi.tuiskula, \\ iliana.ilieva\}@smartinnovationnorway.com
}

\author{
Ferran Torrent, Joan Colomer, Joaquim Meléndez \\ eXiT research group \\ University of Girona \\ Girona, Spain \\ \{ferran.torrent, joan.colomer, joaquim.melendez $\} @$ udg.edu
}

systems, optimization, robust planning.

\section{INTRODUCTION}

stract-Synergies between multiple energy vectors can support decarbonization of local energy islands and, at the same time, relieve stress from the electricity grid. Multi-vector energy systems offer flexibility to integrate intermittent and economic local energy generation. However, the implementation and operation of multi-vector energy systems face technical, societal and business-related challenges. This paper identifies these challenges and proposes different tools to tackle them. These tools are to be developed in the E-LAND project funded by European Union under the $\mathbf{H 2 0 2 0}$ scheme. Special focus of the paper is on developing a method for optimal planning and operation of multi-vector grid. considering trade-off between robustness and optimality. Another novelty of technical tool lies in making scheduling decisions on long-term and short-term storage considering demand response as another flexibility resource. By combining technological, societal and business tools, the project expects to create multi-vector energy systems accepted by citizens and with viable business model.

Index Terms-- Energy community, energy storage, multi-energy
Multiple energy vectors like electricity, heat/cool, and gas have interactions with each other on a system level but have been traditionally managed in a decoupled manner. The concept of multi-energy systems (or energy-hubs) brings operation and planning of various energy vectors under a unified scope. Different energy carriers and sources are often referred to as energy vectors. Studies have shown that interconnecting multiple energy vectors and optimizing them compared to isolated optimization of individual energy vectors [1]-[3]. Such interconnected energy systems have more flexibility than conventional systems as they exploit advantages of different energy vectors [4]. However, challenges exist in practical implementation of such systems at a community, technical and business level. The E-LAND project aims to overcome challenges at all the three levels by developing a modular toolbox for converting today's segregated energy system into an integrated one. By together results in higher renewable energy integration as

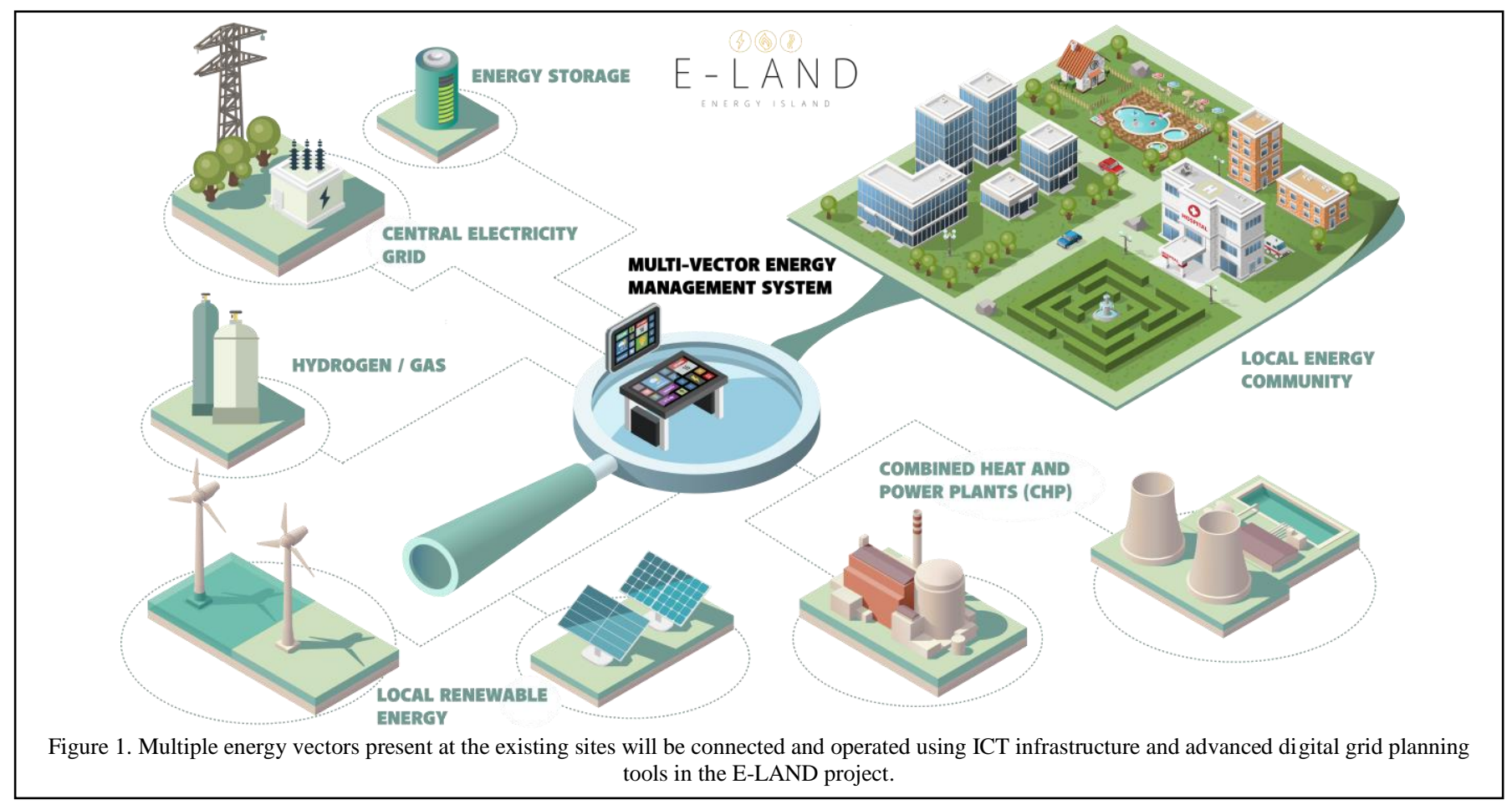


combining multiple vectors at the system level E-LAND tools will decarbonize local energy islands (e.g. isolated villages, small cities, urban districts, rural areas with weak or nonexisting grid connections) in a reliable and secure way. Figure 1 shows the vision of the project, where already existing multiple energy vectors are to be connected using digital technologies.

Modeling and optimization of multi-vector energy systems comes with additional complexity [5]. And the key focus of this paper is to present how the E-LAND project addresses this technical challenge. The paper first describes the ELAND concept followed by the approach towards grid planning and operation of multi-vector energy systems. The last section of the paper concludes with the way forward for the E-LAND project.

\section{E-LAND CONCEPT}

E-LAND brings coherent innovation across three planes: technology, community and business (Figure 2). The final product is a toolbox consisting of tools to build decarbonized, multi-vector energy islands on a foundation of advanced ICT and data analytics technologies, strong community engagement tools and solid business models. The toolbox is modular and customizable to specific local requirements, expandable to incorporate new tools and interoperable with standards-based legacy systems.

Technologically, E-LAND bridges the communication and information flow between different local energy systems to create and implement an integrated solution. Socially, ELAND incorporates citizens and other local community members as part of the solution development team to facilitate bottom-up solution development. Involving citizens to cocreate solutions ensures better acceptance of novel technologies and facilitates changes in behavior, which together strengthen energy reliability and increase the self- sufficiency of communities. Businesswise E-LAND develops tools, which will support market players to innovate their business model and cope with energy-transition and rapid implementation of renewable energies, storage facilities and data-analytic tools. To meet the goals of the project a cocreative process has been designed (Figure 3). To start with, the idea for E-LAND is conceptualized as a solution to a generic problem. During the execution phase, the project is to delve deeper into the pilot specific challenges at all the three planes (i.e., technology, community and business). The execution phase of the project comprises of five steps: i) analyze, ii) define, iii) technology development, iv) test and v) evaluate. There are two feedback loops going back from the testing phase towards problem definition and technology development for further refinement of these steps. The tools are to be implemented and tested at three pilot locations in Europe (Spain, Norway \& Romania) and two simulated pilots in India.

Changes in technology, market structure, regulations and societal needs are inevitable and, thus, new tools need to be constantly developed to address new challenges. Considering this practicality, E-LAND adopts the approach of delivering a toolbox where existing tools can be upgraded to solve new problems when encountered. Market actors can either take the whole toolbox to develop and provide full-scale solutions or select specific tools to further advance their expertise in a particular domain.

\section{A. Technological tools}

E-LAND uses real settings to advance and demonstrate the existing concept of multi-energy systems. The multi-vector energy management system consists of software services and hardware. The three software services to be included are described below:

- Data management service featuring data pre-processing

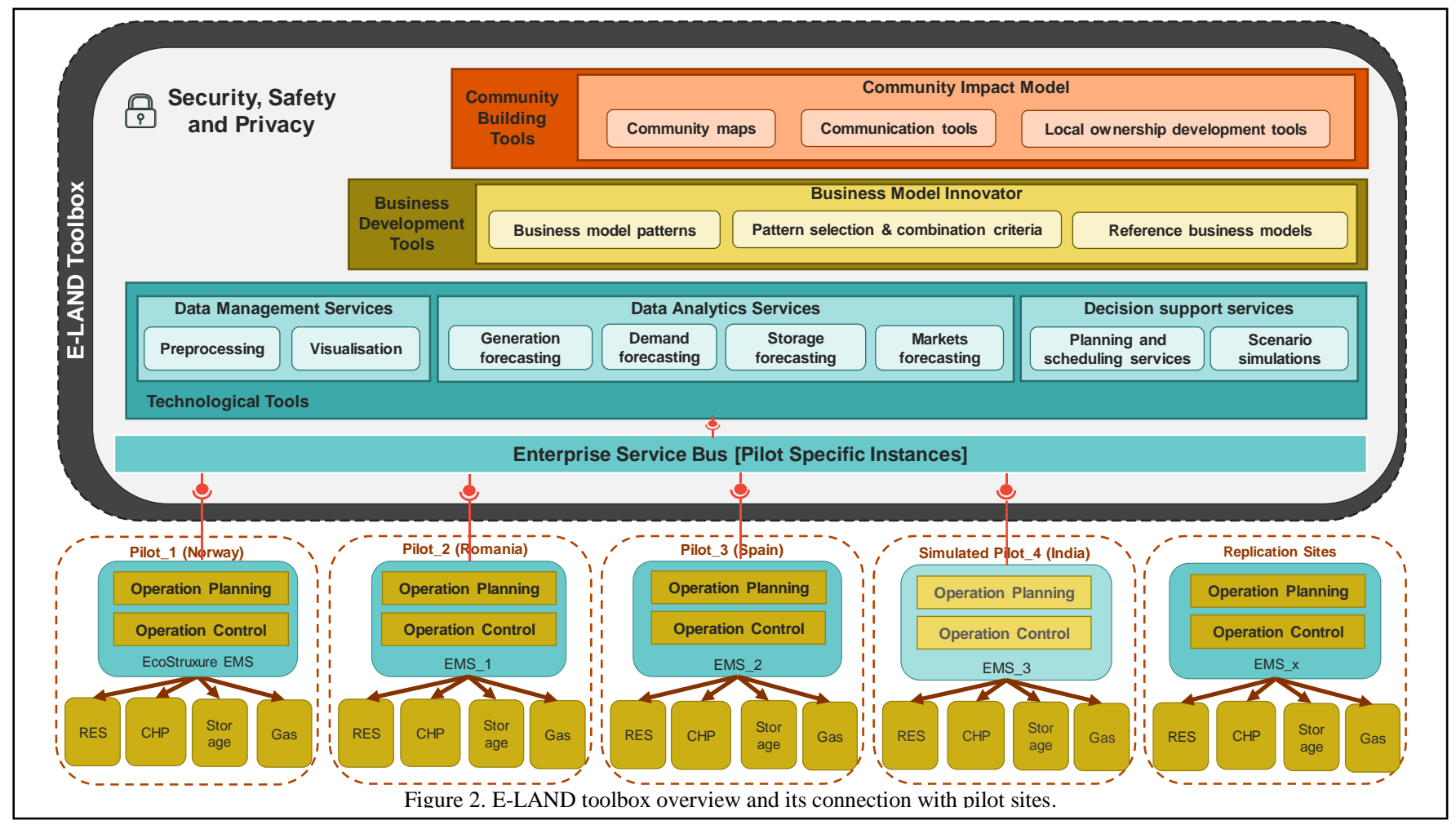


and visualization capabilities. This service loads all production, consumption, and storage data from different energy vectors to a central data system. It enables the exploitation of synergies between different energy vectors.

- Data analytic service providing forecasting for all production, consumption, storage availability and flexibility availability. Forecasting is done using advanced machine learning algorithms and simulations. For each of the above-mentioned resources, there is a separate forecasting tool. Innovatively, the solution enables different Energy Management Systems (EMS) to communicate to each other and form a self-correcting forecasting mechanism.

- Optimization service providing optimal scheduling of multi-vector energy system assets. There are several key innovations in this. Firstly, optimization services connect different energy vectors and optimize them together to improve the hosting capacity of existing infrastructure. Secondly, end-user flexibility is considered as a resource and is scheduled with other generation and storage resources. By storing energy in different vectors, imbalances are considered on a daily, weekly and seasonal basis, always choosing the best option for the system. The third innovation factor is the ability to optimize storage together with end-user flexibility.

Hardware used in E-LAND comprises of communication infrastructure required to fit on top of existing energy infrastructure and is connected to intelligent software tools. This will allow a seamless flow of data and control signals to and from the multi-vector energy management system.

Technology tools of the E-LAND Toolbox are to be modular, expandable and customizable. The software services discarded. The E-LAND Toolbox setup can be adapted as per local requirements.

\section{B. Business tools}

Market actors lack tools that can bring business model innovation to their businesses and this is a major obstacle for further deployment of multi-energy systems. The business tools of E-LAND Toolbox address relevant stakeholders' needs by equipping them with tools to develop new business models for novel energy services, which are relevant to multivector energy islands or communities. Tools to identify viable business model patterns and to subsequently develop sitespecific business cases are developed. The business model tools advance the concept of "business model navigator" for multi-energy systems [6]. Pattern selection and combination framework are developed to induce business model innovation. It is an open toolbox, which can be used by various actors, such as energy communities, DSOs, and energy entrepreneurs to develop business models that suit their ambitions. It is a particular aim of E-LAND to make energy islands commercially self-sustainable. Towards this direction, is to provide evidence of the toolbox's effectiveness through real-life examples. New reference business models are to be created for key stakeholders using the developed tools. Specific attention is given to creating business models for seasonal storage at the community/district level.

\section{Community development tools}

The community tools of the E-LAND Toolbox are to help overcome existing social barriers when introducing new solutions in the energy domain. The project is to deliver tools focused on the development of engagement strategies, and design incentives for different stakeholders present in a community. Tools will be developed in the form of common

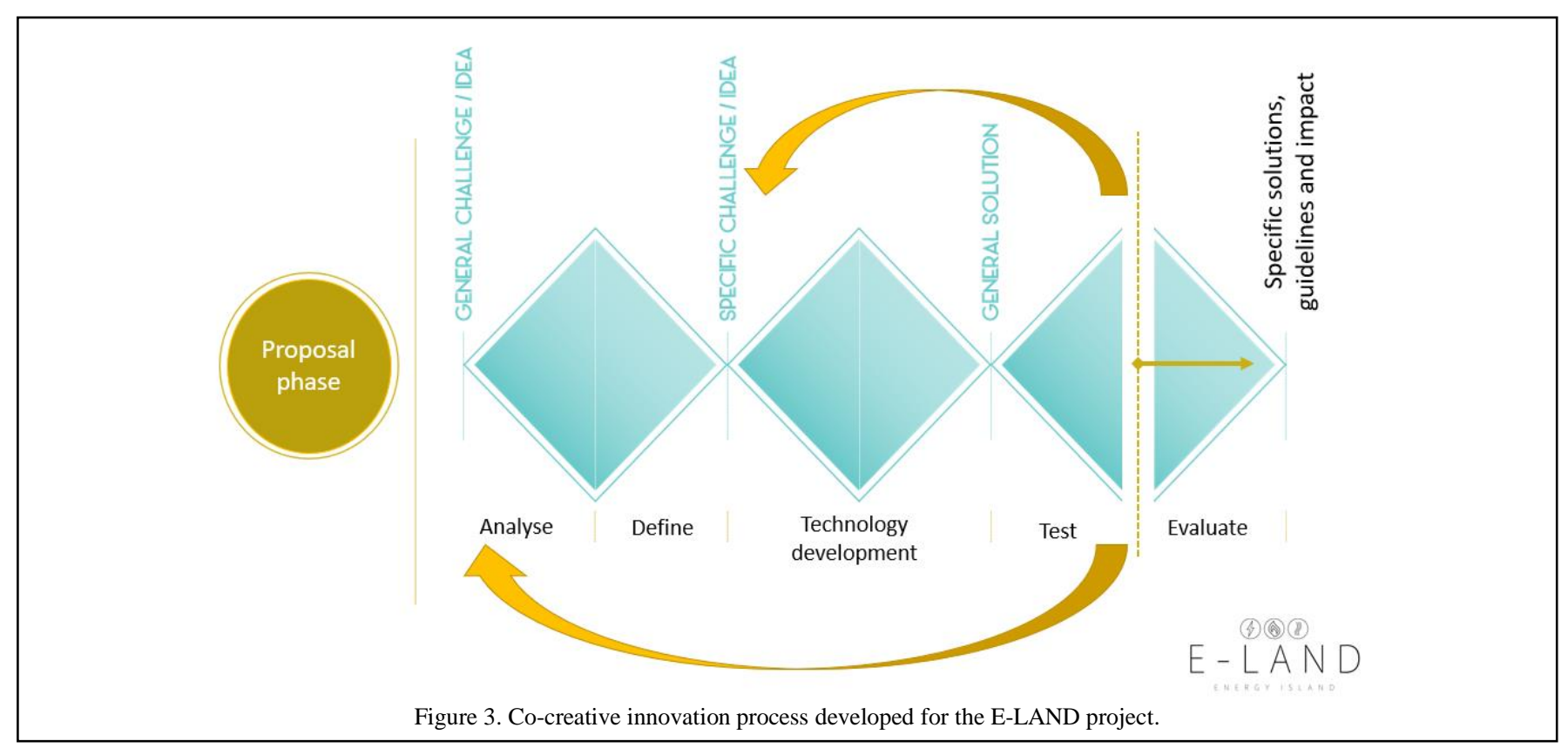

can interoperate with existing platforms and legacy EMS. New modules can be added, and redundant modules can be impact models based upon Ostrom's principles of managing commons and the three elements to determine tipping point 
[7], [8]. These tools are to ensure that the technology is not only accepted but also desired by the end-users. Technologies have been explored, developed and implemented in previous research initiatives, but many of them do not involve citizens to develop solutions and thus, they are challenged with generating permanent impact. End-users in E-LAND are intensively engaged from day one of the project period, starting with mapping of local communities. To create solutions that citizens and other local actors are willing to utilize upon, it is imperative that tools are created in cooperation with the local communities, facilitating their contribution, and not only active participation, but also active leadership. This can help accelerate future innovation processes. Community building tools secure the community's involvement towards the solution and help ensure that technology developed will generate the maximum possible impact expected from it. Thus, these tools support building a strong and aware community.

\section{MULTI-VECTOR GRID PLANNING AND OPERATION}

E-LAND aims at introducing robust multi-vector scheduling for energy islands to ensure energy balance and reliability, while hosting capacity and benefits are maximized even in the presence of stochastic energy sources and nondeterministic energy demand. E-LAND methodology provides decision support to optimize multi-vector operation in a trade-off situation between robustness and optimality, i.e. degree of optimization of the problem objective(s). At the same time, all relevant technological, statutory, contractual, and ecological boundary conditions are taken into consideration in this complex optimization process. For this purpose, an intelligent energy management system (EMS) is further developed and used. Main components that are part of the intelligent multi-vector EMS are the real-time optimization and coordination, the forecasting and the unit commitment modules.
The real-time optimization capabilities of the EMS refer to online optimization and coordination, generation management, energy exchange management, and load management. The module implemented in the project uses innovative and intelligent algorithms to calculate the distribution of the entire corrective power value across the individual generation units, various storage options available and flexible load classes, to determine the new desired operating points for these units.

Forecasting the energy demand from electrical and thermal loads is typically performed as a function of the type of day (e.g. workday or weekend) and time of day. The forecast of renewable energy generation is also important and is based on the weather forecast and the characteristics of the generation units and renewable energy sources. The embedded forecasting modules of EMS allow for parameterizing the forecast bandwidth for the system operator/user to determine the reserve and risk strategies for system operation in advance. Depending on the confidence intervals selected, the width of the forecasting band varies and, accordingly, so does the amount of power to be kept as reserve. In addition, if local online measured weather data is available, a refinement mechanism is used to adjust the imported external weather forecast data with the online data. The refinement mechanism is a sliding correction algorithm that can be parameterized to minimize the difference between the external forecast and the local measured weather data by the current point in time. The resulting internal weather forecast is made available to other EMS planning and optimization functions.

Unit commitment problem refers to the problem of finding the energy production of a set of generators in a defined timehorizon to achieve a particular goal. In the presence of storage systems and demand response, this problem is extended to scheduling the production and/or consumption of all controllable assets.

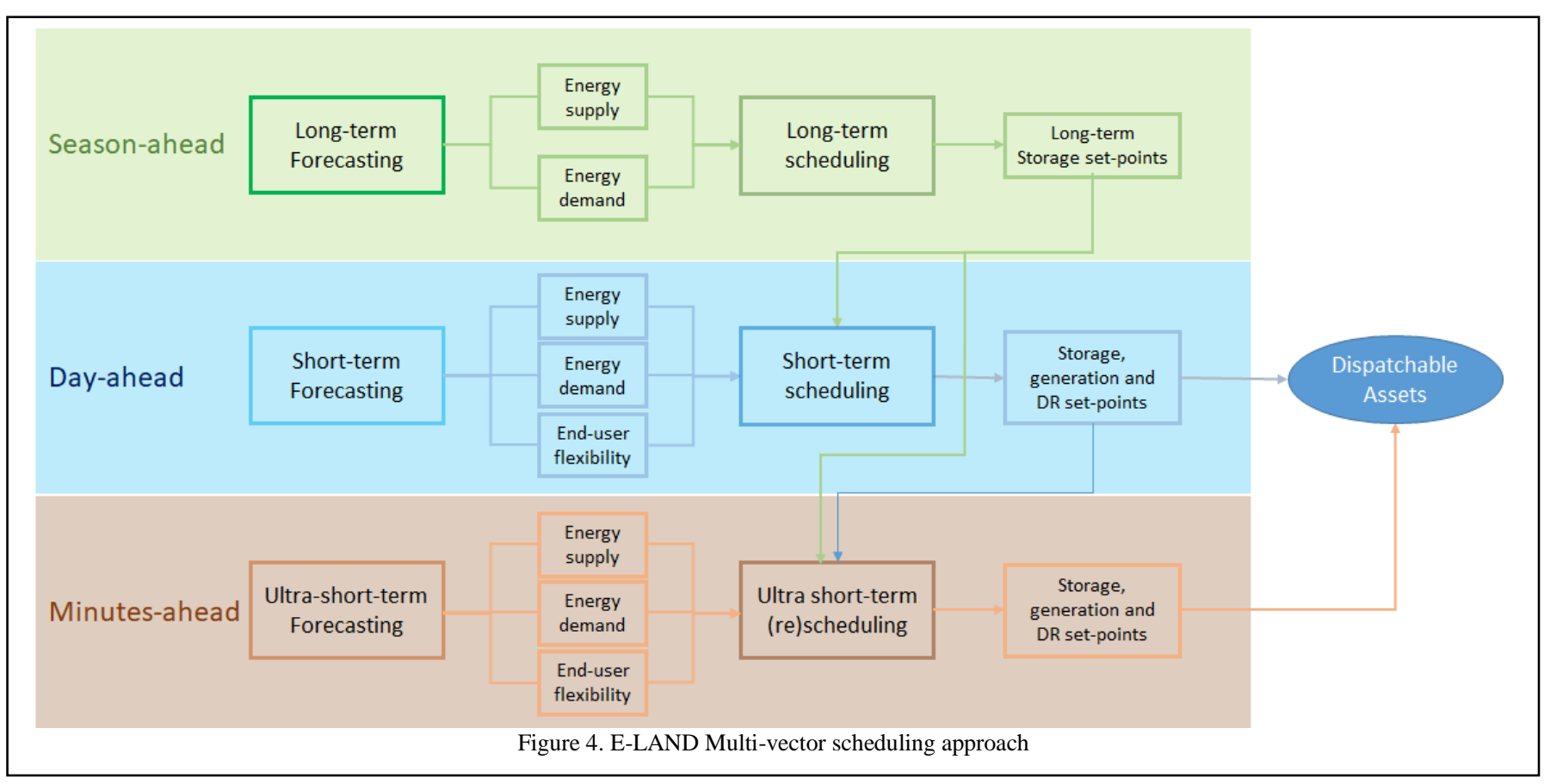




\section{Multi-vector unit commitment problem}

The current shift to a more electrified heating and cooling sector and the proliferation of power-to- $X$ technologies have promoted the inclusion of multi-energy system (or energy hub) in the unit commitment problem. A model of an energy hub is presented in [9] to perform 24-hour-ahead scheduling to maximize profit. The authors in [10] go one step further and propose to tackle the problem of scheduling a grid of interconnected energy hubs.

However, data sources such as energy demand, end-user flexibility or energy production from stochastic energy sources, are forecasts with an associated uncertainty, which should be handled in the scheduling problem. Uncertainty related to wind turbines production or energy price is modelled in respectively [11] and [12] to schedule an energy hub.

The aforementioned research is focused on a particular timehorizon, usually short-term. Nevertheless, energy communities, and especially energy islands, experience great seasonal unbalances which must be tackled. Thus, E-LAND aims to optimize the operation of a multi-energy island like the one illustrated in Figure 1, not only at a short-term horizon, but also at a long-term horizon.

For doing so, various time horizons are considered as depicted in Figure 4. First, long-term scheduling is calculated according to long-term forecast of energy supply and demand. Because of this scheduling, long-term energy storage set-points (or instructions) are derived. These setpoints define energy storage constraints for the short-term scheduling problem and the cost for not fulfilling them. The goal of this phase is to minimize seasonal unbalances by using long-term energy storage systems by prioritizing the use of renewable energy resources, i.e. decarbonize the multienergy system.

Second, short-term scheduling, e.g. day-ahead scheduling, considers long-term scheduling set-points, the forecasted energy demand and supply, and the expected flexibility of the end-users to find an optimal schedule of all controllable assets (i.e. storage systems, controllable generators). The goal of this phase is to match energy demand and supply while long-term set-points are met, and the overall cost and $\mathrm{CO}_{2}$ emissions are minimized. Uncertainty of forecast is tackled in the scheduling process by embedding it in the formulation of the optimization problem and by considering the trade-off between robustness and optimality.

Third, short-term schedules are modified when more accurate energy demand and supply forecasts are available, e.g. 15 minute-ahead. This phase, like the previous one, also considers long-term set-points. Nevertheless, it also considers short-term schedules and modifies them according to the more accurate ultra-short-term forecasts. Note that this phase also provides a short-term schedule, e.g. 24-hour schedule, but in the re-scheduling process, the estimated amount of uncertainty is considered to prioritize the optimization of those time instants with less associated uncertainty.

As the reader may have supposed, the three phases are not run in the same frequency. Season-ahead scheduling is performed a few times per year, while short-term scheduling can be run from one to several times per day. Similarly, shortterm schedules are modified upon ultra-short-term forecasts availability, i.e. every 15 minutes.

The described process is abstract and independent of energy resources and energy storage systems. However, the formulation of the optimization problems models the specificities of each energy system. In this context, E-LAND aims at combined management of the electricity, heat and cooling vectors, with the corresponding storage, supply and demand assets.

Electricity supply is considered stochastic from generators such as PV systems and wind turbines, and controllable when produced by generators or systems such as diesel generators and transformers connected to the main power grid. Heat and cooling supply is also considered stochastic from generators such as solar thermal systems, and controllable from generators such as boilers using natural gas, waste heat pumps, etc. These systems entail the modeling of not just their operational constraints and efficiency, but also uncertainty and time-dependent price of electricity.

Energy demand is considered non-deterministic and, therefore, it is forecast and has an associated uncertainty. Furthermore, energy demand is assumed to have a particular level of flexibility, e.g. HVAC (heating, ventilation and air conditioning) set-points permit some margins.

Energy storage systems can be classified as long-term and short-term storage systems. E-LAND considers short-term energy storage systems such as batteries, electric vehicle, water tanks and reefer containers (for thermal storage). On the other hand, hydrogen production through electrolyzers and hydrogen storage is used as long-term energy storage option.

\section{CONCLUSION}

As the future energy system is going to be more and more distributed, managed by novel business actors and based on variable renewables and storage, the importance of intelligent multiple energy management and optimization tools becomes even more crucial. So far, the solutions available have been focusing mostly on one energy vector such as electricity or heat, whereas E-LAND will develop tools addressing the benefits of managing and optimizing all existing energy vectors including arising vectors such as storage and end-user flexibility to find the best mix to use always. This kind of solution combined with community tools to ensure long-term end-user acceptance and adoption and business tools to secure the financial viability of an energy island, has the potential to transform the way energy is currently produced, transferred, stored and used. Especially for isolated areas with weak or expensive energy infrastructure, the E-LAND toolbox could provide economical solutions for securing the energy supply.

\section{ACKNOWLEDGMENT}

E-LAND project has received funding from the European Union's Horizon 2020 research and innovation programme under grant agreement No 824388 . 


\section{REFERENCES}

[1] E. Ceseña and P. Mancarella, "Operational optimization and environmental assessment of integrated district energy systems," in proc. 2016 IEEE Power Systems Computation Conference (PSCC), (pp. 1-7).

[2] P. Mancarella, "MES (multi-energy systems): An overview of concepts and evaluation models," Energy, vol. 65, pp. 1-17, Feb. 2014.

[3] J. Devlin, K. Li, P. Higgins, and A. Foley, “A multi vector energy analysis for interconnected power and gas systems," Applied energy, 192, pp.315-328, Apr 2017.

[4] E. M. Cesena and P. Mancarella, "Energy Systems Integration in Smart Districts: Robust Optimisation of Multi-Energy Flows in Integrated Electricity, Heat and Gas Networks," IEEE Trans. Smart Grid, Apr 2018.

[5] P. Mancarella, G. Andersson, J.A. Peças-Lopes and K.R. Bell, "Modelling of integrated multi-energy systems: drivers, requirements, and opportunities," in proc.2016 IEEE Power Systems Computation Conference (PSCC) (pp. 1-22).

[6] O. Gassmann, K. Frankenberger, and M. Csik, The business model navigator: 55 models that will revolutionise your business, Pearson UK, 2014.

[7] E. Ostrom, Governing the commons : the evolution of institutions for collective action, Southern Economic Journal, 60(1), 1993, pp.249-251.

[8] M. Gladwell, The tipping point: How little things can make a big difference, Little, Brown, 2006.

[9] I. G. Moghaddam, M. Saniei, and E. Mashhour, "A comprehensive model for self-scheduling an energy hub to supply cooling, heating and electrical demands of a building," Energy, vol. 94, pp. 157-170, Jan. 2016.

[10] H. Yang, T. Xiong, J. Qiu, D. Qiu, and Z. Y. Dong, "Optimal operation of DES/CCHP based regional multi-energy prosumer with demand response," Appl. Energy, vol. 167, pp. 353-365, Apr. 2016.

[11] M. H. Javidi, S. Khaleghi, and M. Zarif, "Assessment of electricity price uncertainty impact on the operation of multi-carrier energy systems," IET Generation, Transmission \& Distribution, vol. 9, no. 16, pp. 2586-2592, Dec. 2015.

[12] A. Najafi, H. Falaghi, J. Contreras, and M. Ramezani, "Medium-term energy hub management subject to electricity price and wind uncertainty," Appl. Energy, vol. 168, pp. 418-433, Apr. 2016. 